\title{
Human viral encephalitis associated with suid herpesvirus 1
}

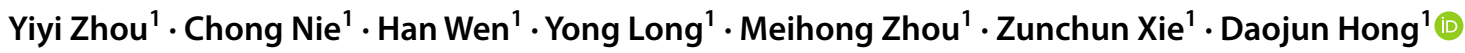

Received: 23 June 2021 / Accepted: 22 September 2021 / Published online: 13 October 2021

(c) Fondazione Società Italiana di Neurologia 2021

\begin{abstract}
Background Suid herpesvirus type 1 (SHV1) is a type of neurotropic virus able to infect various species. However, the clinical cases of human SHV1 encephalitis are still rarely reported, and the clinical characteristics, treatment, and prognosis of human SHV1 encephalitis are still unclear.

Methods In this study, we reported 2 cases of human encephalitis associated with SHV1 infection and reviewed the other 18 cases from the literatures. A total of 20 cases with human SHV1 encephalitis were summarized and re-analyzed.

Results Nineteen of 20 patients had a history of swine-related occupational exposure before illness onset. All patients initially presented with influenza-like symptoms and then developed seizures, disturbed consciousness, and endophthalmitis. All patients with clinical outcome of modified Rankin Scale of 5 or 6 suffered from rapid progressive respiratory failure. The results of cerebrospinal fluid (CSF) indicated aseptic or viral infection. MRI findings of SHV1 encephalitis were prone to distribute in temporal-frontal and insular cortex, which was similar to the pattern of herpes simplex virus encephalitis, while some cases with involvements of gray matter nuclei had a high rate of mortality. Metagenomic next-generation sequencing (mNGS) revealed that all patients had unique SHV1 sequences with variable reads in the CSF.

Conclusions The variant SHV1 can cause a new type of human viral encephalitis, characterized by acute, fulminating, and catastrophic central nervous system infection. Rapid progressive respiratory failure and extensive lesions of deep gray matter nuclei might be indicators to poor prognosis. No approved treatments for the encephalitis are available, but it is possible to diagnose encephalitis quickly by mNGS.
\end{abstract}

Keyword Suid herpesvirus type 1; Pseudorabies virus; Encephalitis; Metagenomic next-generation sequencing

\section{Background}

Suid herpesvirus type 1 (SHV1), also called pseudorabies virus (PRV), is a neurotropic virus that belongs to the Alphaherpesvirinae subfamily and genus Varicellovirus in the family of Herpesviridae [1]. Swine is the primary natural host for SHV1 [2]. The classic viruses, first discovered in swine in 1902, have been associated with fatal encephalitis in piglets, boars sterility, reproductive disorders in sows, and respiratory diseases in growing and fattening pigs and have brought about a great number of economic loss to the swine industry since its global spreading since 1980s [3, 4].

Daojun Hong

hongdaojun@hotmail.com

1 Department of Neurology, The First Affiliated Hospital of Nanchang University, Yong Wai Zheng Street 17\#, Nanchang 330006, Jiangxi Province, China
SHV1 can infect not only swine, but also a wide range of vertebrates including cattle, sheep, cats, dogs, minks, bears, skunks, and mice, indicating that the virus has a strong ability of transmission cross species [5]. Mravak et al. reported 3 patients of suspected human SHV1 infection diagnosed by SHV1 positive antibody without subtype identification [6]. Nevertheless, the disease of human SHV1 infection had been debatable until Ai et al. found some unique SHV1 sequences in the vitreous humor from an endophthalmitis patient through metagenomic next-generation sequencing (mNGS), and the possibility of SHV1 infecting humans across the species barrier was identified at the first time [7]. In the same year, Zhao et al. reported 4 cases of suspected SHV1 encephalitis for the first time based on clinical features and pathogen analysis of cerebrospinal fluid (CSF) by mNGS [8]. However, the cases of human SHV1 encephalitis are still rarely reported, and the clinical characteristics, treatment, and prognosis of encephalitis associated with SHV1 remain unclear. Therefore, we 
reported 2 additional cases of human SHV1 encephalitis and reviewed the other 18 cases reported in the literatures. We retrospectively analyzed the clinical processes of SHV1 encephalitis in order to improve awareness about this new type of human viral encephalitis.

\section{Materials and methods}

\section{Subjects}

The patients were retrospectively collected from neurological intensive care unit in the First Affiliated Hospital of Nanchang University. Initial symptoms, age of onset, occupation, clinical manifestations, treatments, and prognosis were collected from their relatives and medical records. A battery of laboratory examinations including blood chemistry, infectious screening, and immunological screening were conducted in the 2 patients.

\section{Patient consent statement}

All samples and medical data were obtained after a written consent signed by each family in compliance with the bioethical laws of China as well as the Declaration of Helsinki. The research was also approved by the ethic committee of the First Affiliated Hospital of Nanchang University.

\section{Brain MRI}

All magnetic resonance image (MRI) examinations were performed in the 2 patients as routine clinical care through a 3.0 T MR scanner. Conventional T1-weighted spin echo (T1WI) sequences, T2-weighted spin echo (T2WI) sequences, fluid-attenuated inversion recovery (FLAIR) sequences, and diffused weight image (DWI) sequences were conducted according to their own routine procedures. MRI with contrast was conducted at least one time in the 2 patients.

\section{Cerebrospinal fluid NGS}

Five milliliters CSF sample were collected in a sterilized RNase-free container and then stored and delivered at dry ice for a mNGS test. DNA was extracted with QIAamp DNA Microbiome Kit (at\#51,704, QIAGEN, Germany). After enzymatic fragmentation of DNA to a size of 200-300 bp, the sequencing libraries were constructed through end repair, adapter ligation, and amplification. Sequencing templates were prepared by using the OneTouch2 System (Life Technologies, CA, USA) and then sequenced on the BioelectronSeq 4000 sequencer (CapitalBio Corporation, Beijing, China). The original sequencing data is first subjected to data quality control, and the sequences of less than $50 \mathrm{bp}$ and low complexity were removed. The qualified reads were mapped to the human reference genome (GRCh38) using the Burrows-Wheeler Aligner to remove the human sequence. The remaining data were aligned to the NCBI microbial genome database (ftp://ftp.ncbi.nlm.nih.gov/genomes/) for the detection of pathogens. The final pathogen results included a list of suspected pathogens, hitreads, and statistics on the coverage of the genome. All pathogen genome sequences contained 13,992 species of bacteria, 1,659 species of fungi, 13,000 species of viruses, and 287 species of parasites. The reference values, hitreads, and coverage of microbial pathogen were calculated according to the samples of 100 control people. The $5 \%, 75 \%, 95 \%$ quartile, median, and maximum values were obtained, respectively, and then the intervals (5-95\%) were used as the reference range for normal background. Suspected pathogen need to meet the following criteria: (1) hitreads number greater than $95 \%$ quartile of the reference range; (2) the coverage is more than 95\% quartile of the reference range; and (3) suspected targets have potentially pathogenic infection.

To validate the mNGS results, we performed polymerase chain reaction (PCR) analysis and Sanger sequencing on the CSF. The amplification primers are SHV1-gB-F: ACAAGT TCAAGGCCCACATCTAC; SHV1-gB-R: GTCYGTGAA GCGGTTCGTGAT.

\section{Literature review}

We retrieved literatures in multiple databases including PubMed, EMBASE, Scopus, Web of Science, EBSCO, Google Scholar, and Wanfang database using the keywords "pseudorabies virus encephalitis" or "suid herpesvirus encephalitis." The screening protocol was followed a PRISMA workflow. All included cases were required to have SHV1 DNA sequence evidence, and then the clinical characteristics, laboratory results, treatments, complications, and outcomes of all patients were summarized and reanalyzed.

\section{Results}

\section{Clinical features of two patients with SHV1 encephalitis}

\section{Patient 1}

The patient was a 51-year-old woman without specific medical history, but she is a housewife who frequently handles raw pork when cooking. She was transferred to our unit for a critical care with fever for 1 week, delirium, and seizures for 3 days. Initially, the patient had a fever accompanied by headache and drowsiness, but she did not visit doctor since 
the symptoms were considered as a common cold. After 4 days, the patient had abnormal behaviors such as ramblings and tearing the sleeve and then immediately presented with generalized tonic-clonic seizure. The recurrent seizures lasted for 2-3 min and caused the patient in coma. She had respiratory failure due to status epilepticus, and endotracheal intubation was inserted. The patient was clinically diagnosed as encephalitis with status epilepticus, based on no obvious pathological findings on cranial computerized tomography $(\mathrm{CT})$. She was transferred to our neurologic intensive care unit immediately, when her vital signs were stable. On admission, physical examination revealed a sedative state with mechanical ventilator supporting and 6/15 (E1; V1; M4) score of Glasgow coma scale (GCS). The Kernig's, Brudzinski's, and Babinski's signs were all negative, while JOLT test was not performed due to the sedative state.

A routine blood test revealed increased counts of white blood cell (WBC) and neutrophils. Biochemical examination showed a mild elevation of liver enzymes. A battery of serum immunological pathogens was all negative (Supplemental Table 1). Blood cultures were negative to bacteria, tuberculosis, and fungus. The opening pressure of lumber puncture was $220 \mathrm{mmH}_{2} \mathrm{O}$ (normal $80-180 \mathrm{mmH}_{2} \mathrm{O}$ ). CSF analysis showed WBC count of $1 \times 10^{6} / \mathrm{L}$ (normal $0-5 \times 10^{6} / \mathrm{L}$ ), glucose of $3.1 \mathrm{mmol} / \mathrm{L}$ (normal $2.8-4.5 \mathrm{mmol} / \mathrm{L}$ ), chloride of $122.3 \mathrm{mmol} / \mathrm{L}$ (normal $120-132 \mathrm{mmol} / \mathrm{L}$ ), and protein of $340 \mathrm{mg} / \mathrm{L}$ (normal $150-450 \mathrm{mg} / \mathrm{L}$ ). CSF cytology revealed the total count of cells was 80 with lymphocyte
$60 \%$ (48 cells), neutrophils $30 \%$ ( 24 cells), and monocytes $10 \%$ ( 8 cells). The acid-fast staining and India ink staining, as well as bacterial smear and culture, were all negative. CSF and serum were negative to autoimmune encephalitisrelated antibodies available (Supplemental Table 1).

Brain MRI on admission revealed high signals on FLAIR and DWI along the bilateral temporal lobes and right insular cortex (Fig. 1A-E). Brain MRI at 1 month after admission showed diffused lesions involving bilateral temporal-frontalparietal lobes, right occipital lobe, insular cortex, thalamus, basal ganglia, and brain stem with variable enhancements on bilateral temporal lobes, as well as hemorrhagic lesion at the occipital lobe (Fig. 1F-J). On admission, a chest CT scan was performed, but no abnormalities were found (Fig. 2A-B). Electroencephalogram (EEG) showed generalized slow wave (1-2 Hz delta frequency) on the background (Fig. 3A).

Since the clinical features and MRI findings indicated an acute diffused cortical inflammatory involvement, as well as the CSF tests revealed a normal pattern with mild lymphocytic pleocytosis, the patient was clinically suspected as viral encephalitis and immediately was treated with antiviral drugs (acyclovir, $10 \mathrm{mg} / \mathrm{kg}$, q8h), antibiotic (linezolid, $600 \mathrm{mg}$, q12h), antiepileptic (midazolam, $0.05 \mathrm{mg} / \mathrm{kg} / \mathrm{h}$ and sodium valproate, $500 \mathrm{mg}, \mathrm{q} 12 \mathrm{~h}$ ), and decreasing intracranial pressure therapy (mannitol, $25 \mathrm{~g}$ q6h) based on empirical cares. One week after admission, lumbar puncture had been rechecked. The opening pressure of CSF dropped to
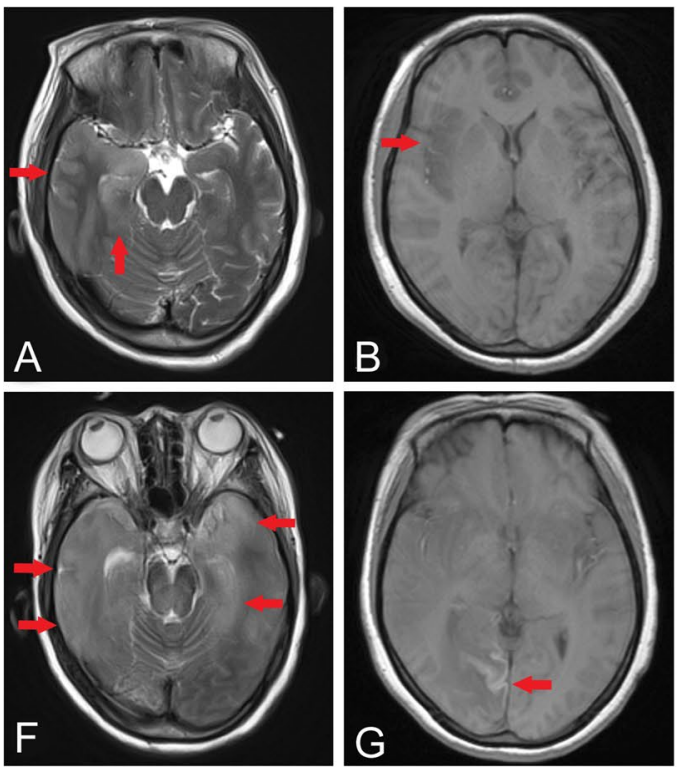
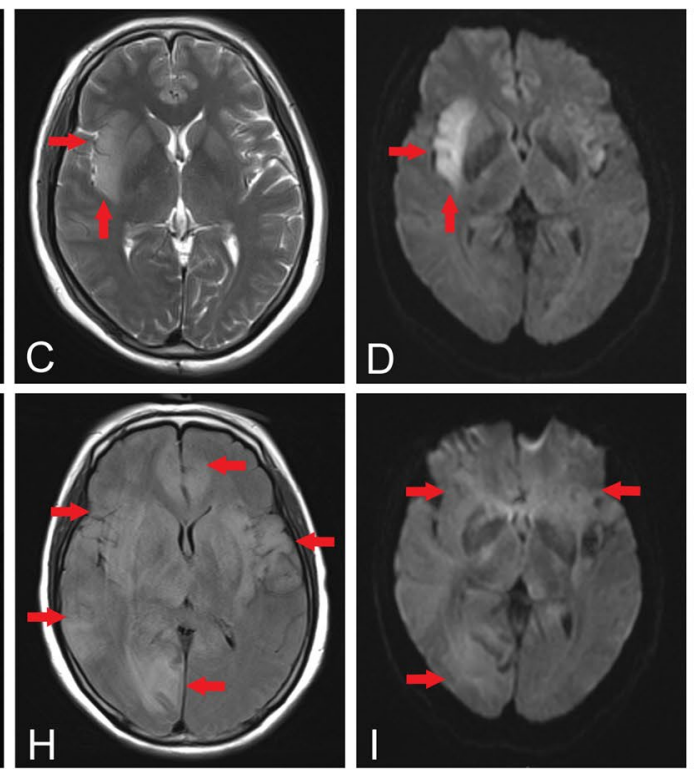
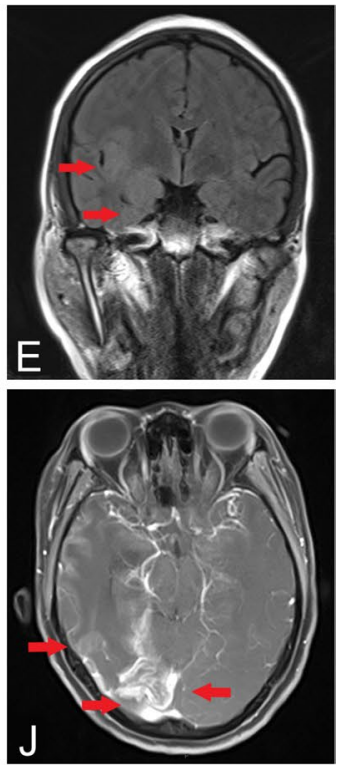

Fig. 1 Dynamic brain MRI changes in patient 1. MRI on admission revealed high intensities in bilateral temporal lobes on T2 (A) and FLAIR (B) and abnormal intensities in the right insular cortex on T1 (C), T2 (D), and DWI (E). MRI at 1 month after admission showed diffused lesions in bilateral temporal lobes and brain stem on
T2 (F); hemorrhagic lesion in occipital lobe on T1 $(\mathbf{G})$; high intensities in temporal-frontal-occipital lobes, thalamus, and basal ganglia on FLAIR (H) and DWI (I); and enhancements on bilateral temporal lobes $(\mathbf{J})$. Arrows indicate the lesions 
Fig. 2 Chest CT scan in the 2 patients. No abnormalities on chest $\mathrm{CT}$ were found in patient 1 on admission ( $\mathbf{A}$ and $\mathbf{B}$ ) and in patient 2 at 3 days after onset (C and D)
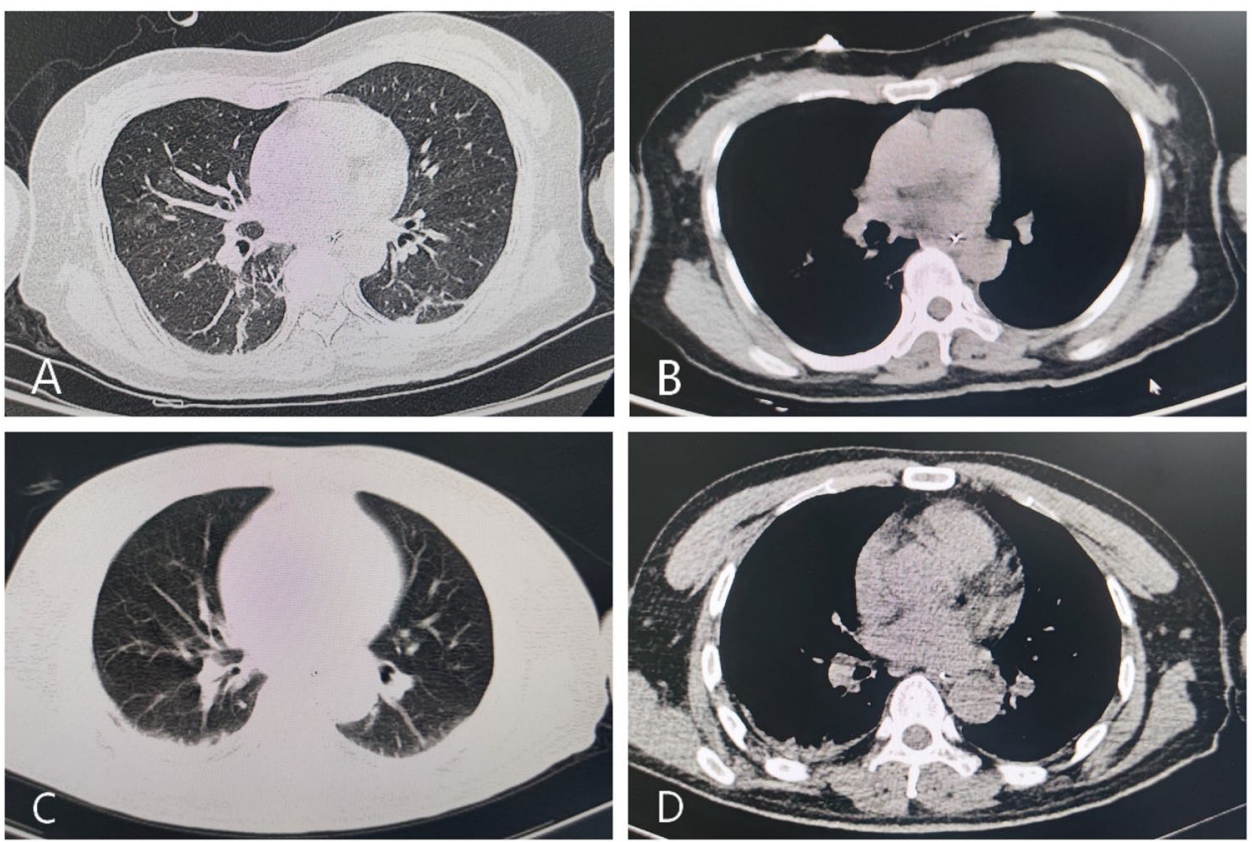

A

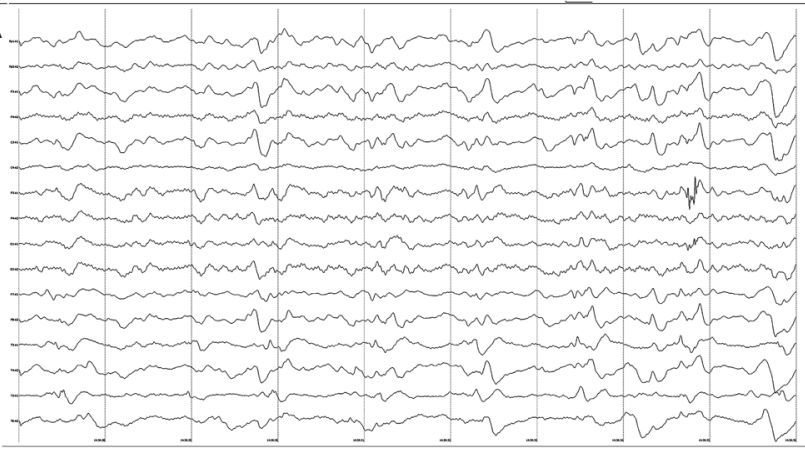

$\mathrm{B}$

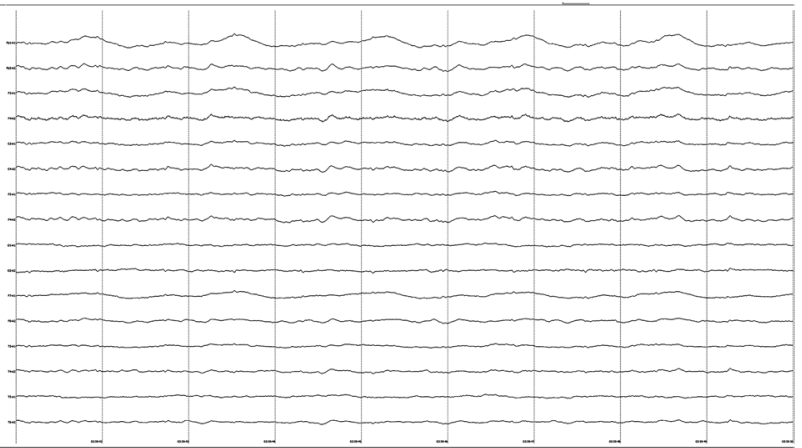

Fig. 3 EEG showed generalized slow wave (1-2 Hz delta frequency) diffusely throughout the background in patient 1 (A); paroxysmal spike wave discharge with diffuse slow wave background in patient 2 (B)

$190 \mathrm{mmH}_{2} \mathrm{O}$; protein level rose significantly to $0.95 \mathrm{mmol} / \mathrm{L}$; glucose was $3.9 \mathrm{mmol} / \mathrm{L}$; chloride was $127.5 \mathrm{mmol} / \mathrm{L}$; and WBC count was $5 \times 10^{6} / \mathrm{L}$. CSF cytology revealed the total count of cells was 180 with lymphocyte $83 \%$ (150 cells), monocytes $10 \%$ (18 cells), neutrophils 5\% (9 cells), and basophils $2.5 \%$ ( 3 cells).

To explore the causative virus, the second CSF sample was sent to mNGS screening and revealed 34 unique reads of suid herpesvirus type 1 with $2.43 \%$ coverage (Fig. 4A). Additionally, Sanger sequencing of a gB segment in SHVI further supported the mNGS result (Fig. 4B). Therefore, the encephalitis was probably associated with SHV1 infection. After a 6-week antiviral treatment, the patient was weaned off ventilator but remained in unconsciousness with metal tracheostomy tube. She was discharged to a local hospital with a modified Rankin Scale (mRS) of 5 and GCS score of 7 (E2, V1, M4).

\section{Patient 2}

A 68-year-old man, a swineherd, was transferred to our clinic due to fever for 4 days and recurrent seizures for 1 day. Initially, the patient had a persistent fever with apathy and was treated as influenza without improvement at the local clinic. Three days later, a chest CT scan was conducted, but no abnormalities were identified (Fig. 2C-D). The patient was transferred to our intensive care unit due to frequent seizures, rapidly progressive status epilepticus and respiratory failure, and then was immediately intubated and treated with sedatives. Physical examination showed medical sedative condition with a GCS score of 3/15(E1; V1; M1). Kernig's and Brudzinski's signs were negative, while the left Babinski's sign was positive.

Routine blood test revealed high counts of WBC and neutrophils. Examinations of blood chemistry were within 

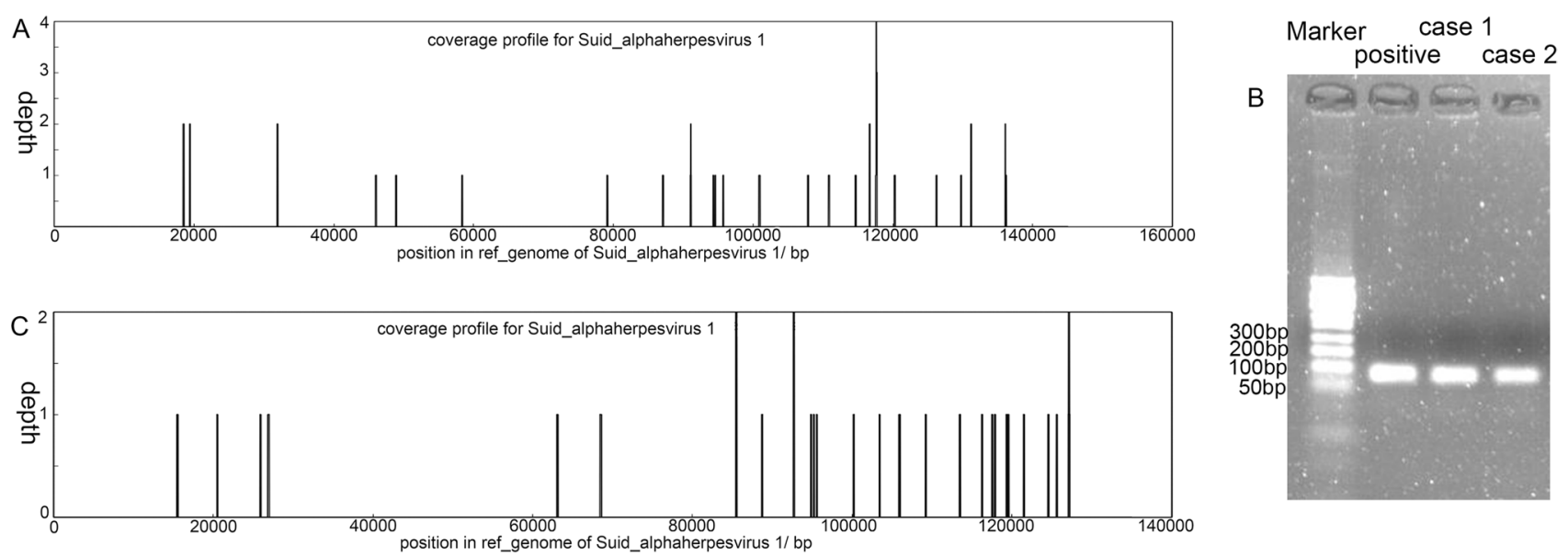
SHV1 further validated the results of mNGS (C)
Fig. $4 \mathrm{mNGS}$ and PCR validation. $\mathrm{mNGS}$ in CSF sample from patient 1 revealed 34 unique reads of SHV1 with $2.43 \%$ coverage (A). mNGS in CSF sample from patient 2 found 29 unique reads of SHV1 with $2.55 \%$ coverage (B). PCR amplification with a 94 bp product of

\section{Summarization of cases with human SHV1 encephalitis reported}

\section{Clinical features and treatment outcome}

pressure of $190 \mathrm{mmH}_{2} \mathrm{O}$. CSF analysis showed WBC count of $20 \times 10^{6} / \mathrm{L}$, glucose of $3.3 \mathrm{mmol} / \mathrm{L}$, chloride of $119 \mathrm{mmol} / \mathrm{L}$, and protein of $1290 \mathrm{mg} / \mathrm{L}$. CSF cytology indicated that the total count of cells were 150 with lymphocyte $60 \%$ (90 cells), neutrophils $20 \%$ (30 cells), monocytes $16 \%$ ( 24 cells), and basophils $4 \%$ ( 6 cells). India ink stain, acid-fast stain, bacterial smear, and antibodies of autoimmune encephalitis were all negative. EEG showed paroxysmal spike wave discharge with diffused slow wave background (Fig. 3B). Based on empirical therapy, the patient was administered with acyclovir $(10 \mathrm{mg} / \mathrm{kg}, \mathrm{q} 8 \mathrm{~h})$, meropenem $(1.0 \mathrm{~g}, \mathrm{q} 8 \mathrm{~h})$, midazolam $(0.05 \mathrm{mg} / \mathrm{kg} / \mathrm{h})$, and sodium valproate (500 $\mathrm{mg}, \mathrm{q} 12 \mathrm{~h}$ ).

Brain MRI on admission showed high signals on FLAIR and DWI along the bilateral temporal-parietal lobes and left insular cortex (Fig. 5A-D), indicating a viral encephalitis. Nevertheless, brain MRI at 10 days after admission showed that the lesions significantly expanded along the temporal-frontal-parietal lobes and thalamus (Fig. 5E-H).

One week later, mNGS screening of CSF sample revealed 29 unique reads of SHV1 with $2.55 \%$ coverage (Fig. 4C). Additionally, Sanger sequencing of a $\mathrm{gB}$ segment in SHV1 further supported the mNGS result (Fig. 4B). Subsequently, intravenous immunoglobulin (IVIG) of $0.4 \mathrm{~g} / \mathrm{kg} /$ days for 5 days were attempted to prevent the disease progression. However, after hospitalization for 3 weeks, the patient still was unconscious and ventilator-dependent and then was palliative care at home. At the end, the patient died 1 week after discharge.
The patient enrollment was depicted in Supplemental Fig. 1. In total, 25 records were identified by the keywords in the multiple databases. After removing the duplication records, 14 of 25 records were screened for cases with SHV1 encephalitis, and then 3 of 14 records were removed due to not clinical reports. Three of 11 records were excluded due to diagnosis ineligibility with evidence insufficiency $(n=2)$ and repeated report $(n=1)$. Finally, 8 of 11 records including 18 cases were qualified to summarize the clinical data of human SHV1 encephalitis.

Besides our study, 18 other cases have been reported as human SHV1 encephalitis with pathogenic evidence [1, 2, 5, 7-12]. All patients came from Mainland China, including 8 cases from Shandong province, 2 cases from Inner Mongolia, 2 cases from Henan province, 2 cases from Hebei province, one case from Hubei province, one case from Guangdong province, one case from Anhui province, and one case from Beijing. Therefore, a total of 20 cases (male, 17; female, 3) of human SHV1 encephalitis were included in the summarization (Table 1 and Supplemental Table 2). $43-52$ years; range 25-63 years). Nineteen of 20 patients worked in industries related to swine, and most of them had a history of occupational exposure before illness onset, such as hand injury or needle stick at work. All patients initially presented with influenza-like symptoms such as fever, headache, and drowsiness and then developed seizures, disturbed consciousness, endophthalmitis, and respiratory failure in
The median age was 50 years (interquartile range (IQR) 

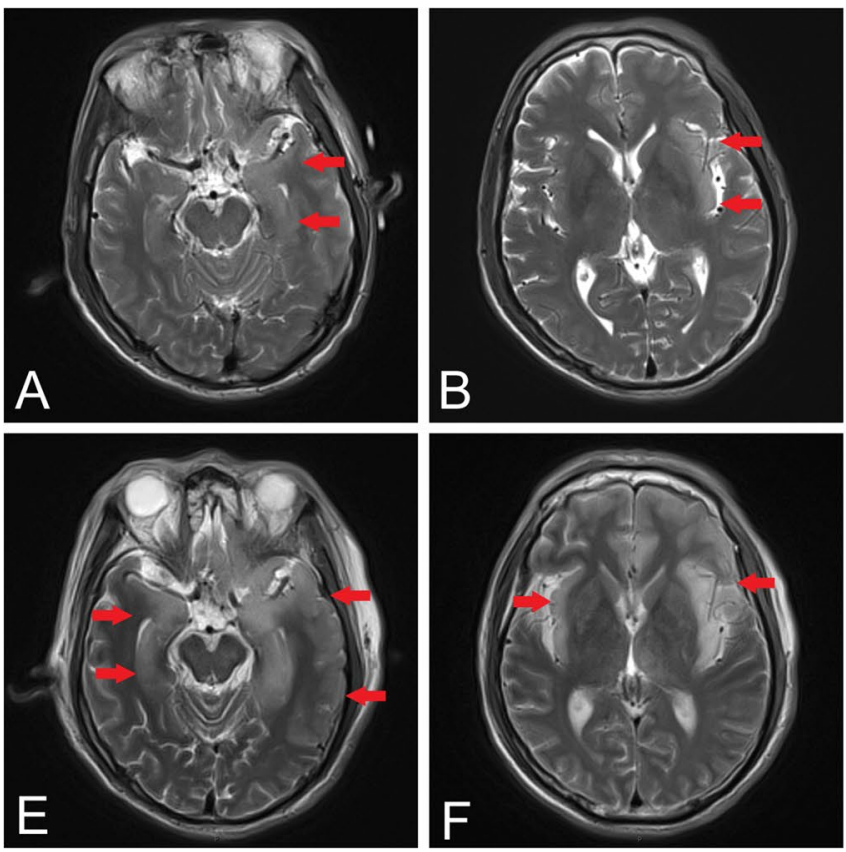

Fig. 5 Dynamic brain MRI changes in patient 2. MRI on admission showed high intensities in bilateral temporal lobes (A) and the left insular cortex (B) on T2 and also in bilateral temporal lobes $(\mathbf{C})$ and the left insular cortex (D) on FLAIR. MRI at 10 days after admission

the first week. The clinical manifestations mainly included fever (20/20), seizures (20/20), disturbed consciousness $(20 / 20)$, respiratory failure $(16 / 20)$, headache $(10 / 20)$, eye involvement (10/20), cognitive impairment (6/20), and psychiatric symptoms (6/20). Pneumonia was the most common complication for SHV1 encephalitis patients, which probably was associated with long-term bedridden and airway dysfunction. Hepatic dysfunction was observed in 3 patients.

A total of 33 times of CSF examinations were conducted in 20 patients (Supplemental Table 3), which showed a median opening pressure of $200 \mathrm{mmH}_{2} \mathrm{O}$ (IQR $\left.167-273 \mathrm{mmH}_{2} \mathrm{O}\right)$, median WBC count of $23 \times 10^{6} / \mathrm{L}(\mathrm{IQR}$ $6-44 \times 10^{6} / \mathrm{L}$ ), and median protein level of $0.5 \mathrm{~g} / \mathrm{L}$ (IQR $0.39-0.67 \mathrm{~g} / \mathrm{L})$. CSF cytology revealed that neutrophils were predominant in the initial stage of the disease in some patients, and lymphocytic pleocytosis with the development of the disease. The level of glucose and chloride in CSF were within normal limits. The findings of CSF indicated the possibility of aseptic encephalitis or viral encephalitis.

Five patients underwent long-term EEG monitoring. All of them had generalized front-temporal slow activity, and three showed epileptiform discharges. These abnormal changes of EEG presumably were associated with impairment of brain function or sedative medicine for seizures.

All patients received antiviral treatment after initial diagnosis with viral encephalitis including acyclovir $(10 \mathrm{mg} / \mathrm{kg}$, q8h) in 17 patients, foscarnet in 6 patients, ganciclovir in 2
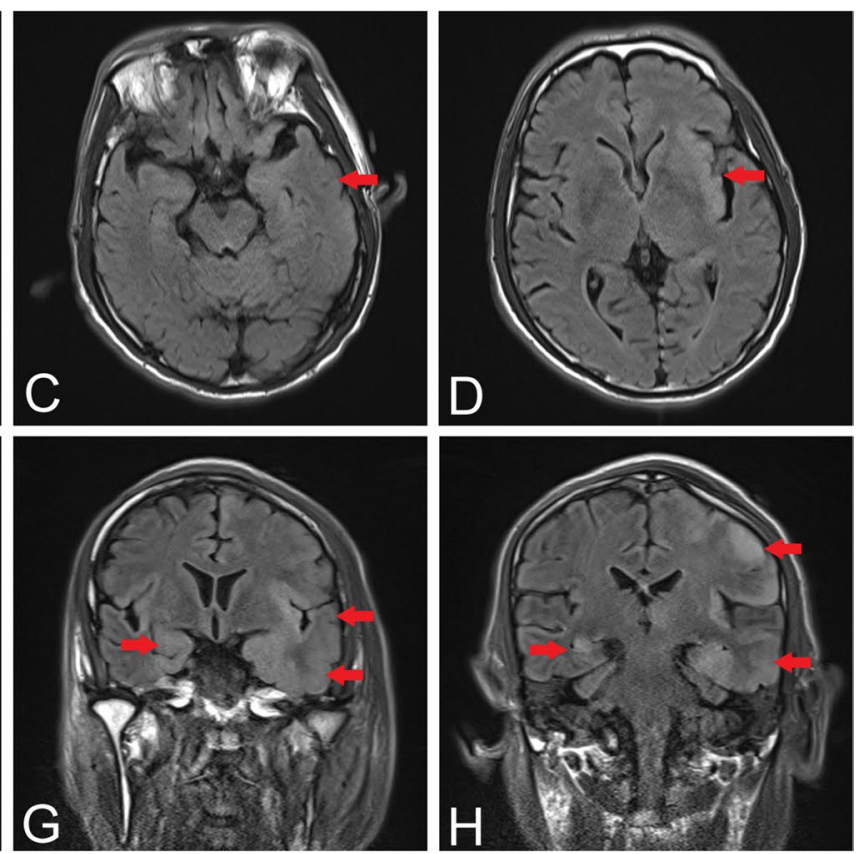

showed that the lesions significantly expanded along the temporalfrontal-parietal lobes and thalamus on T2 (E and F) and FLAIR (G and $\mathbf{H})$. Arrows indicate the lesions

patients, and penciclovir in one patient. Fourteen patients also received immunotherapy including IVIG in 2 patient, glucocorticoids in 3 patients, and both of them in 9 patients. However, the actual dosage of foscarnet, ganciclovir, IVIG, and glucocorticoids were unavailable in most of patients. Most patients showed poor response to acyclovir and immunotherapy and had poor prognosis with a median mRS score of 4 and a mortality rate of $20 \%$ (4/20). Most survivors left severe disability and dysfunction of living capability. Noticeably, antiviral treatments were taken as ganciclovir plus foscarnet in patient 7 , acyclovir plus foscarnet in patient 8 , and penciclovir ( $0.5 \mathrm{~g} /$ day q12h, for 17 days) and foscarnet ( $6 \mathrm{~g} /$ day q12h, for 17 days) in patient 13 , and the 3 patients showed consciousness recovery and relatively good prognosis. All patients needed ICU supporting for a long time (generally more than 1 month), and $76.9 \%$ of patients needed tracheal intubations and ventilator support, which might be associated with a high incidence of status epileptics and respiratory failure in patients with SHV1 encephalitis.

\section{MRI changes}

Nineteen of 20 patients underwent brain MRI, and the remaining one performed a cranial CT scan with no obvious abnormalities. Generally, brain MRI showed high intensity on FLAIR, T2WI, and DWI, as well as low intensity on T1WI. The lesions were distributed at insular lobe in 17 


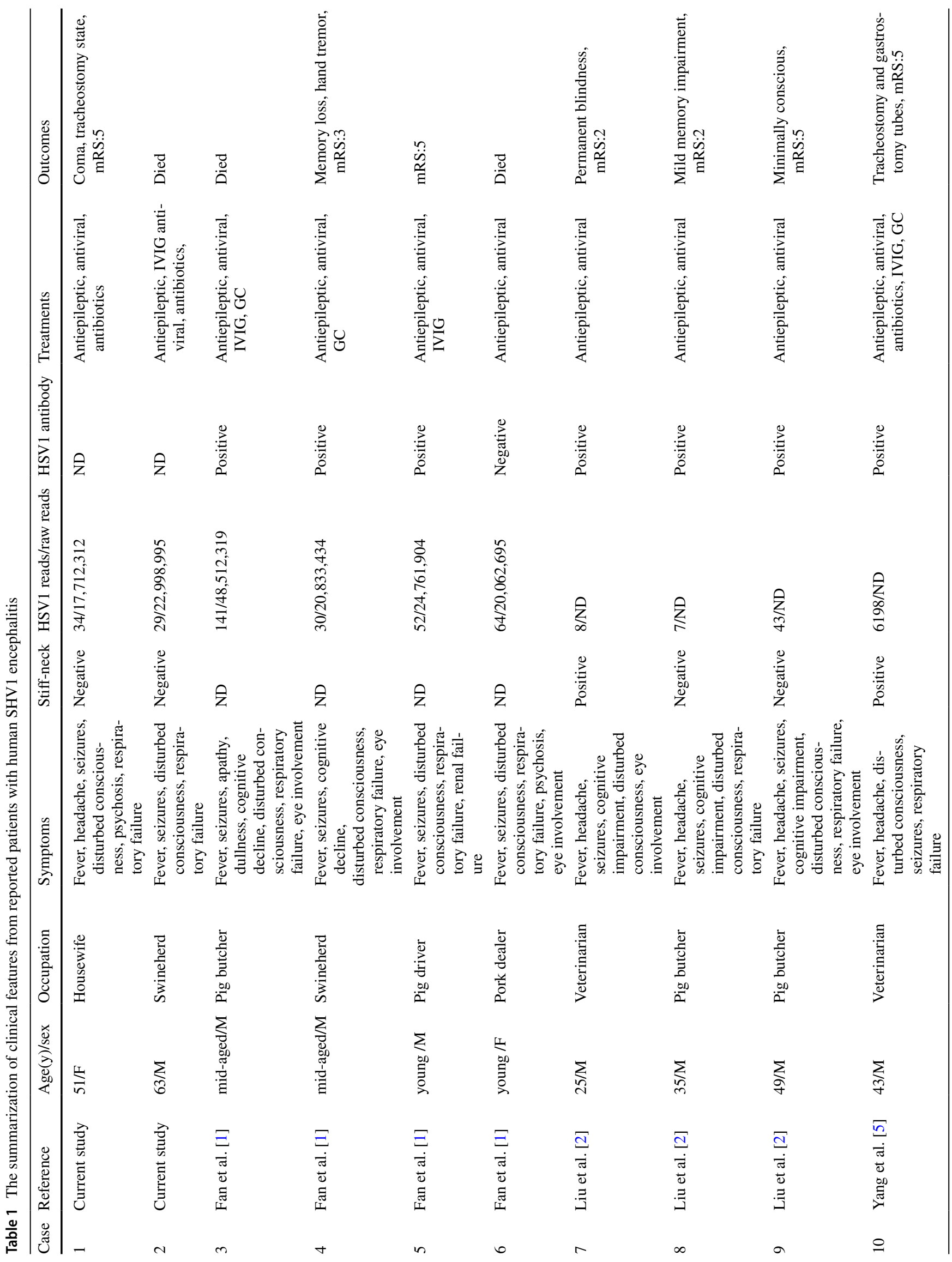




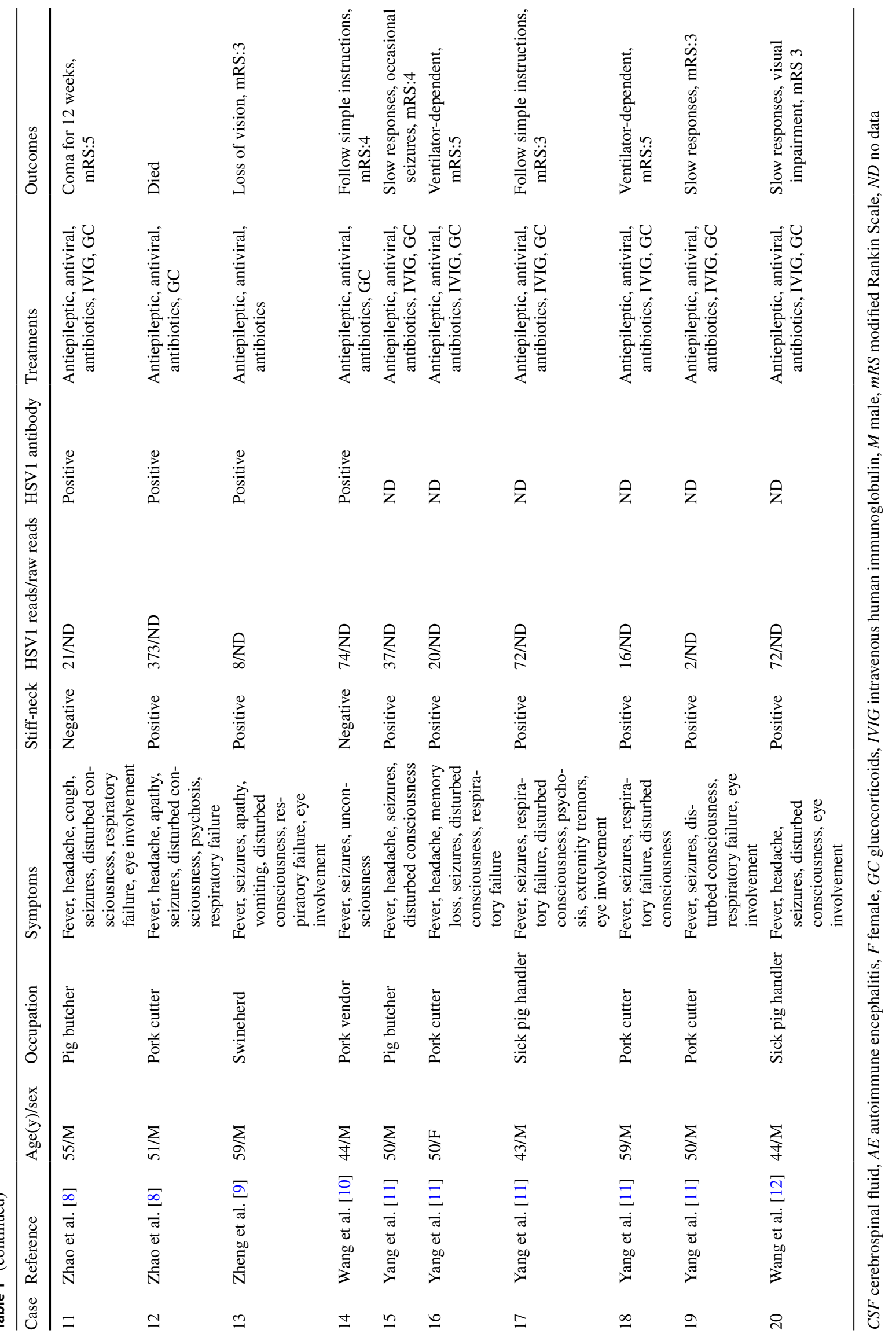


cases, temporal lobe in 17 cases, basal ganglia in 11 cases, frontal lobe in 8 cases, parietal lobe in 3 cases, and brainstem in 3 cases (Supplemental Table 2 and 4). One of our patients had hemorrhagic lesion in the occipital lobe. There were 3 patients with contrast MRI; and 2 patients had linear enhancement along the surface of cortex.

Temporal lobe and insular cortex were found to be the most frequently involved regions in the SHV1 encephalitis. Among the 18 patient with available MRI descriptions, 7 patients just had temporal lobe and/or insular cortex involvement and had clinical outcomes of mean mRS scores of $4.0 \pm 1.3$; the other 11 patients showed additional involvements of cerebral regions including frontal lobe, parietal lobe, occipital lobe, basal ganglia, and brain stem and had clinical outcomes of mean mRS scores of $4.4 \pm 1.4$. There were no significant differences between the 2 groups. Similarly, the mean mRS score was $4.6 \pm 1.5$ in 11 patients with basal ganglia involvement, $3.9 \pm 1.2$ in 7 patients sparing of basal ganglia involvement, and no differences were found between the patients $(p=0.392)$. However, the 4 died patients all had basal ganglia involvement besides diffused cortex involvement, which indicated that patients with involvement of deep gray matter nuclei might have a tendency of poor prognosis.

\section{Microbial analysis}

Microbiological analysis was performed on serum or CSF samples in all patients to identify causative pathogen. India ink stain, acid-fast stain, and bacterial smear test of CSF in all patients showed no abnormalities. mNGS test of CSF were performed on all patients who had unique sequence reads of SHV1. Most patients had reads number between 2 and 373 and low coverage between 0.1 and $16 \%$, while one patient had unique sequence of 6198 reads with $80.58 \%$ of coverage (Table 1). All reads were uniformly distributed in the whole genome region of SHV1. In addition, serum or CSF positive SHV1 IgG antibody was identified in 11 patients.

\section{Discussion}

Suid herpesvirus type 1 is a double-stranded DNA virus with icosahedral symmetric structure [13]. Phylogenetic analysis for the genome sequences shows that classical SHV1 strains from the USA and Europe are categorized as SHV1 genotype I, and variant SHV1 strains from China are categorized as SHV1 genotype II, which might be associated with several outbreaks of SHV1 infection among swineherds in Jiangxi province of China since 2011 [2, 14]. The symptoms of pseudorabies were assumed to be associated with classical SHV1, while the variant SHV1 was considered to be the pathogen of viral endophthalmitis or encephalitis described in this study.

The first descriptions about pseudorabies came from two technicians exposed to contaminated materials in 1914 and showed weakness, restlessness, sore throat, and itching [15]. Additionally in 1987, three patients with seropositive SHV1 antibodies initially developed fever, sweating, weakness, and tiredness and then progressed to dysphagia, paraesthesia, and tinnitus [6]. The clinical symptoms associated with classical SHV1 were very similar to those of rabies that is one of the most lethal diseases known to human, while the clinical courses showed slow but complete recovery. Therefore, SHV1 used to be named pseudorabies virus. However, the clinical features associated with variant SHV1 showed quiet differences from pseudorabies [1, 2]. According to our summarization, the affected patients initially presented with influenza-like symptoms such as fever, headache, and drowsiness and then quickly developed seizures, disturbed consciousness, endophthalmitis, and respiratory failure finally required ICU supporting for a long time. Especially, all patients with mRS score of 5 or 6 presented with rapid progressive respiratory failure, which indicated an unfavorable prognosis. Therefore, the encephalitis associated with variant SHV1 presented with severe disable or lethal viral encephalitis.

Viral glycoprotein gD of SHV1 mediates the entry process into host cell through binding the host cell by the nectin-1 receptor, similar to herpes simplex viruses (HSV) [16]. Therefore, the MRI lesions of variant SHV1 encephalitis are prone to distribute in temporal-frontal lobes and insular cortex similar to those in HSV encephalitis [10], but some severe cases with disease progression have lesion expansions to parietal-occipital lobes, thalamus, basal ganglia, and brain stem, which are rarely observed in the HSV encephalitis. Although the involved lesions of cerebral regions were not associated with the disease severity in our summarization, we found that the 4 dead patients all had involvements of deep gray matter nuclei besides diffused cortical lesions, which might play an indicator to poor prognosis. Additionally, the hemorrhagic lesions were used to be considered as a differentiated point between SHV1 and HSV encephalitis [11], but our SHV1 encephalitis patient also showed hemorrhagic lesion in the advanced stage of disease.

mNGS is a revolutionary method in the identification of potential pathogens including rare and novel viruses [17]. Currently, mNGS tests of CSF were conducted in all 20 patients, and then unique sequence reads uniformly distributed in the whole genome region of SHV1 were identified; consequently, the diagnosis of SHV1 encephalitis can be supported based on molecular evidence. The first human case of SHV1 infection with mNGS result was a pig farmer who was diagnosed as viral endophthalmitis with fever, headaches, and visual impairment [7]. The mNGS 
showed that the most likely pathogen was variant SHV1, which belonged to genotype II according to the phylogenetic analysis of the SHV1 genome sequence. Next, a patient of SHV1 encephalitis had 6198 unique sequence reads covering $80.58 \%$ of the SHV 1 genome region, also indicating the genotype II of SHV1 $[2,5]$. The other 19 patient with lower coverage of SHV1 genome presented with a very similar phenotype to the patient with high coverage. Therefore, we speculated that all 20 patients with SHV1 encephalitis were associated with the SHV1 genotype II, though available mNGS data were not enough to identify the strain of SHV1 genotype II.

At present, there is no available treatment guideline for SHV1 encephalitis treatment. According to the Clinical Practice Guidelines of Encephalitis by the Infectious Diseases Society of America (IDSA), acyclovir is recommended (I level, A evidence) for herpes virus infection [18]. Therefore, 17 of 20 patients were given full-dose acyclovir, but the patients showed poor response to the conventional antiviral therapy. Studies about piscidin 1 and resveratrol have provided some alternative measures for SHV1 infection, but clinical trials need to be carried out in the future $[19,20]$. Intriguingly, some SHV1 encephalitis patient showed an improvement of consciousness after immediate administration of both penciclovir or ganciclovir plus foscarnet sodium treatment [2,9]. The prompt antiviral treatment might attenuate severe SHV1 encephalitis and facilitate the clinical recovery.
Additionally, antiepileptic, respiratory support, nutritional support, and pneumonia treatment are also important to improve the prognosis in patients with SHV1 encephalitis [12]. Future studies to establish the optimal treatment regimen for SHV1 encephalitis are urgently needed.

According to the guidelines of viral encephalitis [21, 22] and clinical features of the 20 patients with SHV1 encephalitis, we made a proposal for diagnostic workflow of human SHV1 encephalitis (Table 2). (1) Patient should have at least an epidemiological history of occupations related to swine, or history of direct contact with raw pork, or location in SHV1 epidemic area. (2) Patient should have a fever and one symptom of acute brain parenchyma dysfunctions that manifested recurrent seizures/ status epilepticus or severe disturbance of consciousness including coma, delirium, decorticate state, minimally conscious state and vegetative state, or mental disorder. Additionally, acute vision loss due to endophthalmitis with fundus hemorrhage and papilledema could be considered as one of clinical features. (3) Patient had abnormal EEG, aseptic/viral encephalitis in CSF, and abnormal multiple cerebral MRI lesions similar to HSV encephalitis but more involvements of deep gray matter nuclei. (4) Patient should be identified with specific sequences of SHV1 in CSF or blood by mNGS or PCR and positive SHV1 antibody in serum or CSF. The definite diagnosis of SHV1 encephalitis should include an epidemiological history, typical clinical features, abnormal auxiliary examinations, and definite

Table 2 Criteria for clinical diagnosis of human SHV1 encephalitis

\begin{tabular}{|c|c|}
\hline Variables & Descriptions \\
\hline A: Epidemiology one or more $1-3$ & $\begin{array}{l}\text { 1. History of direct contact with raw pork } \\
\text { 2. Occupations related to swine } \\
\text { 3. Location in SHV1 epidemic area }\end{array}$ \\
\hline B: Cardinal symptoms $1+$ one or more $2-5$ & $\begin{array}{l}\text { 1. Fever ( }>37.5^{\circ} \mathrm{C} \text { in mouth) } \\
\text { 2. Recurrent seizures or status epilepticus } \\
\text { 3. Severe disturbance of consciousness (coma, delirium, decorticate state, minimally conscious } \\
\text { state, and vegetative state) } \\
\text { 4. Acute vision loss due to endophthalmitis with fundus hemorrhage and papilledema } \\
\text { 5. Psychiatric symptoms }\end{array}$ \\
\hline C: Auxiliary examinations & $\begin{array}{l}\text { 1. EEG: generalized or predominantly front-temporal slow activity; periodic lateralized epilepti- } \\
\text { form discharges } \\
\text { 2. CSF: consistent with the features of CSF of aseptic encephalitis or viral encephalitis } \\
\text { 3. Brain MRI: high signals in front-temporal cortex, insular lobe, basal ganglia, and brain stem on } \\
\text { FLAIR }\end{array}$ \\
\hline D: Virological tests & $\begin{array}{l}\text { 1. Specific sequences of SHV1 identified in CSF or blood by mNGS or PCR } \\
\text { 2. Positive SHV1 antibody in serum or CSF }\end{array}$ \\
\hline Definite diagnosis & $\mathrm{A}+\mathrm{B}+\mathrm{C}+\mathrm{D} 1$ \\
\hline Probable diagnosis & $\mathrm{A}+\mathrm{B}+\mathrm{C} 1+\mathrm{C} 2+\mathrm{D} 1$ \\
\hline Possible diagnosis & $\mathrm{A}+\mathrm{B}+\mathrm{C}+\mathrm{D} 2$ \\
\hline
\end{tabular}

SHV1 suid herpesvirus type 1, EEG electroencephalogram, CSF cerebrospinal fluid, FLAIR fluid-attenuated inversion recovery, $m N G S$ metagenomic next-generation sequencing 
SHV1 DNA evidence. The detailed workflow can be found in the Table 2.

This study had some limitations that need to be explicitly acknowledged. First, it was a retrospective study; thus, some clinical data in our cases were incomplete that would lower the clinical significance; for example, the SHV1 antibodies were not measured due to lack of serum sample; therefore, it might weaken the diagnosis of SHV1 encephalitis. Second, the descriptions about reported cases were incomplete, so some important clinical features and laboratory results might have bias in the summarization. Third, the diagnostic workflow of human SHV1 encephalitis was just formulated on literature review and our own experiences, so the reliability of diagnostic protocols was undermined and need to be validated in a perspective observation including more cases.

\section{Conclusion}

The variant SHV1, as a zoonotic pathogen, has been associated with a new type of human viral encephalitis characterized by acute, fulminating, and catastrophic central nervous system infection. The rapid progressive respiratory failure may be one of causes to the poor prognosis. Diffused lesions involved in extensive cortex and deep gray matter may also indicate an unfavorable prognosis. Since the underlying pathological pathway for SHV1 in humans is limited, there is no available proven treatment for the encephalitis, though a rapid diagnosis is becoming possible through mNGS.

Supplementary Information The online version contains supplementary material available at https://doi.org/10.1007/s10072-021-05633-0.

Acknowledgements The authors would like to appreciate the patients and their families for their enthusiasm and participation in this study.

Author contribution ZY draft manuscript and analysis of data. WH, $\mathrm{NC}$, and LY contributed to the acquisition and analysis of data. ZM and $\mathrm{XZ}$ contributed to critical revision of the manuscript. HD contributed the study design and drafting the manuscript.

Funding The work was supported by the National Natural Science Foundation of China (No. 81460199) and Double thousand talents program of Jiangxi province (jxsq2019101021).

Availability of data and material All relevant data are described within the paper. Deidentified data can be requested. Data can be requested by all interested researchers, who can be contacted via the corresponding author.

\section{Declarations}

Ethical approval The research was approved by ethics committee of the first affiliated hospital of Nanchang University.

Consent for publication Not Applicable.
Conflict of interest The authors declare no competing interests.

\section{References}

1. Fan S, Yuan H, Liu L et al (2020) Pseudorabies virus encephalitis in humans: a case series study. J Neurovirol 26(4):556-564

2. Liu Q, Wang X, Xie C et al (2020) A novel human acute encephalitis caused by pseudorabies virus variant strain. Clin Infect Dis. https://doi.org/10.1093/cid/ciaa987

3. Hanson RP (1954) The history of pseudorabies in the United States. J Am Vet Med Assoc 124(925):259-261

4. Pomeranz LE, Reynolds AE, Hengartner CJ (2005) Molecular biology of pseudorabies virus: impact on neurovirology and veterinary medicine. Microbiol Mol Biol Rev 69(3):462

5. Yang H, Han H, Wang H, Cui Y, Liu H, Ding S (2019) A case of human viral encephalitis caused by pseudorabies virus infection in China. Front Neurol 10:534

6. Mravak S, Bienzle U, Feldmeier H, Hampl H, Habermehl KO (1987) Pseudorabies in man. Lancet 1(8531):501-502

7. Ai JW, Weng SS, Cheng Q et al (2018) Human endophthalmitis caused by pseudorabies virus infection, China, 2017. Emerg Infect Dis 24(6):1087-1090

8. Zhao WL, Wu YH, Li HF et al (2018) Clinical experience and next-generation sequencing analysis of encephalitis caused by pseudorabies virus. Zhonghua Yi Xue Za Zhi 98(15):1152-1157

9. Zheng L, Liu X, Yuan D et al (2019) Dynamic cerebrospinal fluid analyses of severe pseudorabies encephalitis. Transbound Emerg Dis 66(6):2562-2565

10. Wang D, Tao X, Fei M et al (2020) Human encephalitis caused by pseudorabies virus infection: a case report. J Neurovirol 26(3):442-448

11. Yang X, Guan H, Li C et al (2019) Characteristics of human encephalitis caused by pseudorabies virus: a case series study. Int J Infect Dis 87:92-99

12. Wang Y, Nian H, Li Z, Wang W, Wang X, Cui Y (2019) Human encephalitis complicated with bilateral acute retinal necrosis associated with pseudorabies virus infection: a case report. Int $\mathrm{J}$ Infect Dis 89:51-54

13. Guan X, Liu J, Jiang H, Wu CX, Chen HC, Liu ZF (2018) Expression of pseudorabies virus-encoded long noncoding RNAs in epithelial cells and neurons. J Neurovirol 24(5):597-605

14. Wong G, Lu J, Zhang W, Gao GF (2019) Pseudorabies virus: a neglected zoonotic pathogen in humans. Emerg Microbes Infect $8(1): 150-154$

15. Von Ratz ST (1914) Die empfaglichkeit der tier fur paralysis bulbaris infectiosa. Zeitschrift Infectionskran-kheiten 15:2

16. Li A, Lu G, Qi J et al (2017) Structural basis of nectin-1 recognition by pseudorabies virus glycoprotein D. PLoS Pathog 13(5):e1006314

17. Guan H, Shen A, Lv X et al (2016) Detection of virus in CSF from the cases with meningoencephalitis by next-generation sequencing. J Neurovirol 22(2):240-245

18. Tunkel AR, Glaser CA, Bloch KC et al (2008) The management of encephalitis: clinical practice guidelines by the Infectious Diseases Society of America. Clin Infect Dis 3:3

19. Zhao X, Cui Q, Fu Q et al (2017) Antiviral properties of resveratrol against pseudorabies virus are associated with the inhibition of IкB kinase activation. Sci Rep 7(1):8782

20. Hu H, Guo N, Chen S et al (2019) Antiviral activity of Piscidin 1 against pseudorabies virus both in vitro and in vivo. Virol $\mathrm{J}$ 16(1):95

21. Solomon T, Michael BD, Smith PE et al (2012) Management of suspected viral encephalitis in adults-Association of British 
Neurologists and British Infection Association National Guidelines. J Infect 64(4):347-473

22. Venkatesan A, Geocadin RG (2014) Diagnosis and management of acute encephalitis: a practical approach. Neurol Clin Pract 4(3):206-215
Publisher's note Springer Nature remains neutral with regard to jurisdictional claims in published maps and institutional affiliations. 